1 Hacettepe Journal of Mathematics and Statistics

ก Volume 43 (2) (2014), $197-208$

\title{
An introduction to fuzzy soft topological spaces
}

\author{
Abdülkadir Aygünoğlu* Vildan Çetkin ${ }^{\dagger}$ Halis Aygün ${ }^{\ddagger \S}$
}

\begin{abstract}
The aim of this study is to define fuzzy soft topology which will be compatible to the fuzzy soft theory and investigate some of its fundamental properties. Firstly, we recall some basic properties of fuzzy soft sets and then we give the definitions of cartesian product of two fuzzy soft sets and projection mappings. Secondly, we introduce fuzzy soft topology and fuzzy soft continuous mapping. Moreover, we induce a fuzzy soft topology after given the definition of a fuzzy soft base. Also, we obtain an initial fuzzy soft topology and give the definition of product fuzzy soft topology. Finally, we prove that the category of fuzzy soft topological spaces FSTOP is a topological category over SET.
\end{abstract}

Keywords: fuzzy soft set, fuzzy soft topology, fuzzy soft base, initial fuzzy soft topology, product fuzzy soft topology.

2000 AMS Classification: 06D72, 54A05, 54A40.

\section{Introduction}

Most of the existing mathematical tools for formal modeling, reasoning and computing are crisp, deterministic, and precise in character. But, in real life situation, the problems in economics, engineering, environment, social science, medical science etc. do not always involve crisp data. For this reason, we cannot successfully use the traditional classical methods because of various types of uncertainties presented in these problems. To exceed these uncertainties, some kinds of theories were given like theory of fuzzy sets [21], intuitionistic fuzzy sets [4], rough sets [16],i.e., which we can use as mathematical tools for dealing with uncertainties. But all these theories have their inherent difficulties as what were pointed out by Molodtsov in [15]. The reason for these difficulties is, possibly, the inadequacy of the parametrization tool of the theories. Consequently, Molodtsov [15] initiated the concept of soft set theory as a new mathematical tool for dealing with vagueness and uncertainties which is free from the above difficulties.

\footnotetext{
*Department of Mathematics, University of Kocaeli, Umuttepe Campus, 41380, Kocaeli TURKEY Email: abdulkadir.aygunoglu@kocaeli.edu.tr

$\dagger$ Email: vildan.cetkin@kocaeli.edu.tr

¥ Email: halis@kocaeli.edu.tr

${ }^{\S}$ Corresponding Author.
} 
Applications of Soft Set Theory in other disciplines and real life problems are now catching momentum. Molodtsov [15] successfully applied the soft set theory into several directions, such as smoothness of functions, game theory, Riemann integration, Perron integration, theory of measurement, and so on. Maji et al. [14] gave first practical application of soft sets in decision making problems. They have also introduced the concept of fuzzy soft set, a more generalized concept, which is a combination of fuzzy set and soft set and also studied some of its properties. Ahmad and Kharal $[2,11]$ also made further contributions to the properties of fuzzy soft sets and fuzzy soft mappings. Soft set and fuzzy soft set theories have a rich potential for applications in several directions, a few of which have been shown by some authors [15, 18].

The algebraic structure of soft set and fuzzy soft set theories dealing with uncertainties has also been studied by some authors. Aktaş and Çağman [3] have introduced the notion of soft groups. Jun [7] applied soft sets to the theory of BCK/BCI-algebras, and introduced the concept of soft BCK/BCI algebras. Jun and Park [8] and Jun et al. [9, 10] reported the applications of soft sets in ideal theory of BCK/BCI-algebras and $d$-algebras. Feng et al. [6] defined soft semirings and several related notions to establish a connection between soft sets and semirings. Sun et al. [20] presented the definition of soft modules and construct some basic properties using modules and Molodtsov's definition of soft sets. Aygünoğlu and Aygün [5] introduced the concept of fuzzy soft group and in the meantime, discussed some properties and structural characteristic of fuzzy soft group.

In this study, we consider the topological structure of fuzzy soft set theory. First of all, we give the definition of fuzzy soft topology $\tau$ which is a mapping from the parameter set $E$ to $[0,1]^{\widetilde{X, E)}}$ which satisfies the three certain conditions. With respect to this definition the fuzzy soft topology $\tau$ is a fuzzy soft set on the family of fuzzy soft sets $\widetilde{(X, E)}$. Also, since the value of a fuzzy soft set $f_{A}$ under the mapping $\tau_{e}$ gives the degree of openness of the fuzzy soft set with respect to the parameter $e \in E, \tau_{e}$ can be thought as a fuzzy soft topology in the sense of Šostak [19]. In this manner, we introduce fuzzy soft cotopology and give the relations between fuzzy soft topology and fuzzy soft cotopology. Then we define fuzzy soft base. Moreover, we induce a fuzzy soft topology by using a fuzzy soft base on the same set. Also, we obtain an initial fuzzy soft topology and then we give the definition of product fuzzy soft topology. Finally, we show that the category of fuzzy soft topological spaces FSTOP is a topological category over SET with respect to the forgetful functor.

\section{Preliminaries}

Throughout this paper, $X$ refers to an initial universe, $E$ is the set of all parameters for $X, I^{X}$ is the set of all fuzzy sets on $X$ (where, $I=[0,1]$ ) and for $\lambda \in[0,1], \bar{\lambda}(x)=\lambda$, for all $x \in X$.

2.1. Definition. $[2,13] f_{A}$ is called a fuzzy soft set on $X$, where $f$ is a mapping from $E$ into $I^{X}$,i.e., $f_{e} \triangleq f(e)$ is a fuzzy set on $X$, for each $e \in A$ and $f_{e}=\overline{0}$, if $e \notin A$, where $\overline{0}$ is zero function on $X$. $f_{e}$, for each $e \in E$, is called an element of the fuzzy soft set $f_{A}$. 
$\widetilde{(X, E)}$ denotes the collection of all fuzzy soft sets on $X$ and is called a fuzzy soft universe $([13])$.

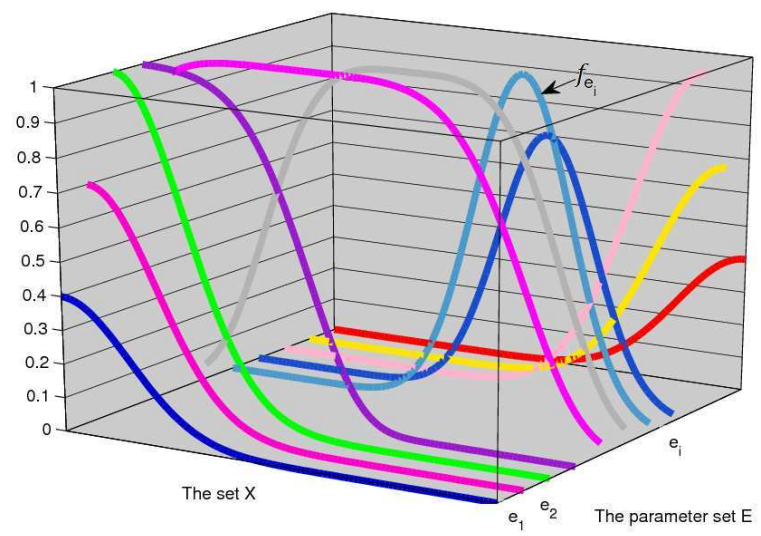

Figure 1. A fuzzy soft set $f_{E}$

2.2. Definition. [13] For two fuzzy soft sets $f_{A}$ and $g_{B}$ on $X$, we say that $f_{A}$ is a fuzzy soft subset of $g_{B}$ and write $f_{A} \sqsubseteq g_{B}$ if $f_{e} \leq g_{e}$, for each $e \in E$.

2.3. Definition. [13] Two fuzzy soft sets $f_{A}$ and $g_{B}$ on $X$ are called equal if $f_{A} \sqsubseteq g_{B}$ and $g_{B} \sqsubseteq f_{A}$.

2.4. Definition. [13] Union of two fuzzy soft sets $f_{A}$ and $g_{B}$ on $X$ is the fuzzy soft set $h_{C}=f_{A} \sqcup g_{B}$, where $C=A \cup B$ and $h_{e}=f_{e} \vee g_{e}$, for each $e \in E$. That is, $h_{e}=f_{e} \vee \overline{0}=f_{e}$ for each $e \in A-B, h_{e}=\overline{0} \vee g_{e}=g_{e}$ for each $e \in B-A$ and $h_{e}=f_{e} \vee g_{e}$, for each $e \in A \cap B$.

2.5. Definition. [2,13] Intersection of two fuzzy soft sets $f_{A}$ and $g_{B}$ on $X$ is the fuzzy soft set $h_{C}=f_{A} \sqcap g_{B}$, where $C=A \cap B$ and $h_{e}=f_{e} \wedge g_{e}$, for each $e \in E$.

2.6. Definition. The complement of a fuzzy soft set $f_{A}$ is denoted by $f_{A}^{c}$, where $f^{c}: E \longrightarrow I^{X}$ is a mapping given by $f_{e}^{c}=\overline{1}-f_{e}$, for each $e \in E$.

Clearly $\left(f_{A}^{c}\right)^{c}=f_{A}$.

2.7. Definition. [13] (Null fuzzy soft set) A fuzzy soft set $f_{E}$ on $X$ is called a null fuzzy soft set and denoted by $\Phi$, if $f_{e}=\overline{0}$, for each $e \in E$.

2.8. Definition. (Absolute fuzzy soft set) A fuzzy soft set $f_{E}$ on $X$ is called an absolute fuzzy soft set and denoted by $\widetilde{E}$, if $f_{e}=\overline{1}$, for each $e \in E$. Clearly $(\widetilde{E})^{c}=\Phi$ and $\Phi^{c}=\widetilde{E}$.

2.9. Definition. ( $\lambda$-absolute fuzzy soft set) A fuzzy soft set $f_{E}$ on $X$ is called a $\lambda$-absolute fuzzy soft set and denoted by $\widetilde{E}^{\lambda}$, if $f_{e}=\bar{\lambda}$, for each $e \in E$. Clearly, $\left(\widetilde{E}^{\lambda}\right)^{c}=\widetilde{E}^{1-\lambda}$.

2.10. Proposition. [2] Let $\Delta$ be an index set and $f_{A}, g_{B}, h_{C},\left(f_{A}\right)_{i} \triangleq\left(f_{i}\right)_{A_{i}},\left(g_{B}\right)_{i} \triangleq$ $\left(g_{i}\right)_{B_{i}} \in \widetilde{(X, E)}, \forall i \in \Delta$, then we have the following properties: 
(1) $f_{A} \sqcap f_{A}=f_{A}, \quad f_{A} \sqcup f_{A}=f_{A}$.

(2) $f_{A} \sqcap g_{B}=g_{B} \sqcap f_{A}, \quad f_{A} \sqcup g_{B}=g_{B} \sqcup f_{A}$.

(3) $f_{A} \sqcup\left(g_{B} \sqcup h_{C}\right)=\left(f_{A} \sqcup g_{B}\right) \sqcup h_{C}, \quad f_{A} \sqcap\left(g_{B} \sqcap h_{C}\right)=\left(f_{A} \sqcap g_{B}\right) \sqcap h_{C}$.

(4) $f_{A}=f_{A} \sqcup\left(f_{A} \sqcap g_{B}\right), \quad f_{A}=f_{A} \sqcap\left(f_{A} \sqcup g_{B}\right)$.

(5) $f_{A} \sqcap\left(\bigsqcup_{i \in \Delta}\left(g_{B}\right)_{i}\right)=\bigsqcup_{i \in \Delta}\left(f_{A} \sqcap\left(g_{B}\right)_{i}\right)$.

(6) $f_{A} \sqcup\left(\prod_{i \in \Delta}\left(g_{B}\right)_{i}\right)=\prod_{i \in \Delta}\left(f_{A} \sqcup\left(g_{B}\right)_{i}\right)$.

(7) $\Phi \sqsubseteq f_{A} \sqsubseteq \widetilde{E}$.

(8) $\left(f_{A}^{c}\right)^{c}=f_{A}$.

(9) $\left(\prod_{i \in \Delta}\left(f_{A}\right)_{i}\right)^{c}=\bigsqcup_{i \in \Delta}\left(f_{A}\right)_{i}^{c}$.

(10) $\left(\bigsqcup_{i \in \Delta}\left(f_{A}\right)_{i}\right)^{c}=\prod_{i \in \Delta}\left(f_{A}\right)_{i}^{c}$.

(11) If $f_{A} \sqsubseteq g_{B}$, then $g_{B}^{c} \sqsubseteq f_{A}^{c}$.

2.11. Definition. $[5,11]$ Let $\varphi: X \longrightarrow Y$ and $\psi: E \longrightarrow F$ be two mappings, where $E$ and $F$ are parameter sets for the crisp sets $X$ and $Y$, respectively. Then the pair $\varphi_{\psi}$ is called a fuzzy soft mapping from $\widetilde{(X, E)}$ into $\widetilde{(Y, F)}$ and denoted by $\varphi_{\psi}: \widetilde{(X, E)} \longrightarrow \widetilde{(Y, F)}$.

2.12. Definition. $[5,11]$ Let $f_{A}$ and $g_{B}$ be two fuzzy soft sets over $X$ and $Y$, respectively and let $\varphi_{\psi}$ be a fuzzy soft mapping from $\widetilde{(X, E)}$ into $\widetilde{(Y, F)}$.

(1) The image of $f_{A}$ under the fuzzy soft mapping $\varphi_{\psi}$, denoted by $\varphi_{\psi}\left(f_{A}\right)$, is the fuzzy soft set on $Y$ defined by $\varphi_{\psi}\left(f_{A}\right)=\varphi(f)_{\psi(A)}$, where

$$
\varphi(f)_{k}(y)= \begin{cases}\bigvee_{x \in \varphi^{-1}(y)}\left(\bigvee_{a \in \psi^{-1}(k) \cap A} f_{a}(x)\right), & \text { if } \varphi^{-1}(y) \neq \emptyset, \psi^{-1}(k) \cap A \neq \emptyset ; \\ 0, & \text { otherwise. }\end{cases}
$$

$\forall k \in F, \forall y \in Y$.

(2) The pre-image of $g_{B}$ under the fuzzy soft mapping $\varphi_{\psi}$, denoted by $\varphi_{\psi}^{-1}\left(g_{B}\right)$, is the fuzzy soft set on $X$ defined by $\varphi_{\psi}^{-1}\left(g_{B}\right)=\varphi^{-1}(g)_{\psi^{-1}(A)}$, where

$$
\varphi^{-1}(g)_{a}(x)=\left\{\begin{array}{ll}
g_{\psi(a)}(\varphi(x)), & \text { if } \psi(a) \in B ; \\
0, & \text { otherwise. }
\end{array}, \quad \forall a \in E, \forall x \in X .\right.
$$

If $\varphi$ and $\psi$ is injective (surjective), then $\varphi_{\psi}$ is said to be injective (surjective).

2.13. Definition. Let $\varphi_{\psi}$ be a fuzzy soft mapping from $\widetilde{(X, E)}$ into $\widetilde{(Y, F)}$ and $\varphi_{\psi^{*}}^{*}$ be a fuzzy soft mapping from $\widetilde{(Y, F)}$ into $\widetilde{(Z, K)}$. Then the composition of these mappings from $\widetilde{(X, E)}$ into $\widetilde{(Z, K)}$ is defined as follows: $\varphi_{\psi^{*}}^{*} \circ \varphi_{\psi} \triangleq\left(\varphi^{*} \circ \varphi\right)_{\psi^{*} \circ \psi}$, where $\psi: E \longrightarrow F$ and $\psi^{*}: F \longrightarrow K$.

2.14. Proposition. [11] Let $X$ and $Y$ be two universes $f_{A},\left(f_{A}\right)_{1},\left(f_{A}\right)_{2},\left(f_{A}\right)_{i} \in$ $\widetilde{(X, E)}, g_{B},\left(g_{B}\right)_{1},\left(g_{B}\right)_{2},\left(g_{B}\right)_{i} \in \widetilde{(Y, F)} \forall i \in \Delta$, where $\Delta$ is an index set, and $\varphi_{\psi}$ be a fuzzy soft mapping from $\widetilde{(X, E)}$ into $\widetilde{(Y, F)}$.

(1) If $\left(f_{A}\right)_{1} \sqsubseteq\left(f_{A}\right)_{2}$, then $\varphi_{\psi}\left(\left(f_{A}\right)_{1}\right) \sqsubseteq \varphi_{\psi}\left(\left(f_{A}\right)_{2}\right)$.

(2) If $\left(g_{B}\right)_{1} \sqsubseteq\left(g_{B}\right)_{2}$, then $\varphi_{\psi}^{-1}\left(\left(g_{B}\right)_{1}\right) \sqsubseteq \varphi_{\psi}^{-1}\left(\left(g_{B}\right)_{2}\right)$.

(3) $f_{A} \sqsubseteq \varphi_{\psi}^{-1}\left(\varphi_{\psi}\left(f_{A}\right)\right)$, the equality holds if $\varphi_{\psi}$ is injective.

(4) $\varphi_{\psi}\left(\varphi_{\psi}^{-1}\left(f_{A}\right)\right) \sqsubseteq f_{A}$, the equality holds if $\varphi_{\psi}$ is surjective. 
(5) $\varphi_{\psi}\left(\bigsqcup_{i \in \Delta}\left(f_{A}\right)_{i}\right)=\bigsqcup_{i \in \Delta} \varphi_{\psi}\left(\left(f_{A}\right)_{i}\right)$.

(6) $\varphi_{\psi}\left(\prod_{i \in \Delta}\left(f_{A}\right)_{i}\right) \sqsubseteq \prod_{i \in \Delta} \varphi_{\psi}\left(\left(f_{A}\right)_{i}\right)$, the equality holds if $\varphi_{\psi}$ is injective.

(7) $\varphi_{\psi}^{-1}\left(\bigsqcup_{i \in \Delta}\left(g_{B}\right)_{i}\right)=\bigsqcup_{i \in \Delta} \varphi_{\psi}^{-1}\left(\left(g_{B}\right)_{i}\right)$.

(8) $\varphi_{\psi}^{-1}\left(\prod_{i \in \Delta}\left(g_{B}\right)_{i}\right)=\prod_{i \in \Delta} \varphi_{\psi}^{-1}\left(\left(g_{B}\right)_{i}\right)$.

(9) $\varphi_{\psi}^{-1}\left(g_{B}^{c}\right)=\left(\varphi_{\psi}^{-1}\left(g_{B}\right)\right)^{c}$.

(10) $\varphi_{\psi}^{-1}\left(\widetilde{E}_{Y}\right)=\widetilde{E}_{X}, \quad \varphi_{\psi}^{-1}\left(\Phi_{Y}\right)=\Phi_{X}$.

(11) $\varphi_{\psi}\left(\widetilde{E}_{X}\right)=\widetilde{E}_{Y}$ if $\varphi_{\psi}$ is surjective.

(12) $\varphi_{\psi}\left(\Phi_{X}\right)=\Phi_{Y}$.

2.15. Definition. (Cartesian product of two fuzzy soft sets) Let $X_{1}$ and $X_{2}$ be nonempty crisp sets. $f_{A} \in\left(\widetilde{X_{1}, E_{1}}\right)$ and $g_{B} \in\left(\widetilde{X_{2}, E_{2}}\right)$. The cartesian product $f_{A} \times g_{B}$ of $f_{A}$ and $g_{B}$ is defined by $(f \times g)_{A \times B}$, where, for each $(e, \mathrm{f}) \in E_{1} \times E_{2}$,

$(f \times g)_{(e, \mathrm{f})}(x, y)=f_{e}(x) \wedge g_{\mathrm{f}}(y)$, for all $(x, y) \in X \times Y$.

According to this definition the fuzzy soft set $(f \times g)_{A \times B}$ is a fuzzy soft set on $X_{1} \times X_{2}$ and the universal parameter set is $E_{1} \times E_{2}$.

2.16. Definition. Let $\left(f_{A}\right)_{1} \times\left(f_{A}\right)_{2}$ be a fuzzy soft set on $X_{1} \times X_{2}$. The projection mappings $\left(p_{q}\right)_{i}, i \in\{1,2\}$, are defined as follows:

$\left(p_{q}\right)_{i}\left(\left(f_{A}\right)_{1} \times\left(f_{A}\right)_{2}\right)=p_{i}\left(f_{1} \times f_{2}\right)_{q_{i}\left(A_{1} \times A_{2}\right)}=\left(f_{A}\right)_{i}$ where $p_{i}: X_{1} \times X_{2} \longrightarrow X_{i}$ and $q_{i}: E_{1} \times E_{2} \longrightarrow E_{i}$ are projection mappings in classical meaning.

\section{Fuzzy soft topological spaces}

To formulate our program and general ideas more precisely, recall first the concept of fuzzy topological space, that is of a pair $(X, \tau)$ where $X$ is a set and $\tau: I^{X} \longrightarrow I$ is a mapping (satisfying some axioms) which assigns to every fuzzy subset of $X$ the real number, which shows "to what extent" this set is open. According to this idea a fuzzy topology $\tau$ is a fuzzy set on $I^{X}$. This approach has lead us to define fuzzy soft topology which is compatible to the fuzzy soft theory. By our definition, a fuzzy soft topology is a fuzzy soft set on the set of all fuzzy soft sets $\widetilde{(X, E)}$ which denotes "to what extent" this set is open according to the parameter set.

3.1. Definition. A mapping $\tau: E \longrightarrow[0,1]^{(\widetilde{X, E)}}$ is called a fuzzy soft topology on $X$ if it satisfies the following conditions for each $e \in E$.

(O1) $\tau_{e}(\Phi)=\tau_{e}(\widetilde{E})=1$

(O2) $\tau_{e}\left(f_{A} \sqcap g_{B}\right) \geq \tau_{e}\left(f_{A}\right) \wedge \tau_{e}\left(g_{B}\right), \quad \forall f_{A}, g_{B} \in \widetilde{(X, E)}$.

(O3) $\tau_{e}\left(\bigsqcup_{i \in \Delta}\left(f_{A}\right)_{i}\right) \geq \bigwedge_{i \in \Delta} \tau_{e}\left(\left(f_{A}\right)_{i}\right), \forall\left(f_{A}\right)_{i} \in \widehat{(X, E)}, i \in \Delta$.

A fuzzy soft topology is called enriched if it provides that

$(\mathrm{O} 1)^{\prime} \tau_{e}\left(\widetilde{E}^{\lambda}\right)=1$.

Then the pair $\left(X, \tau_{E}\right)$ is called a fuzzy soft topological space. The value $\tau_{e}\left(f_{A}\right)$ is interpreted as the degree of openness of a fuzzy soft set $f_{A}$ with respect to parameter $e \in E$. 
Let $\tau_{E}^{1}$ and $\tau_{E}^{2}$ be fuzzy soft topologies on $X$. We say that $\tau_{E}^{1}$ is finer than $\tau_{E}^{2}\left(\tau_{E}^{2}\right.$ is coarser than $\left.\tau_{E}^{1}\right)$, denoted by $\tau_{E}^{2} \sqsubseteq \tau_{E}^{1}$, if $\tau_{e}^{2}\left(f_{A}\right) \leq \tau_{e}^{1}\left(f_{A}\right)$ for each $e \in E, f_{A} \in \widetilde{(X, E)}$.

Example Let $\mathcal{T}$ be a fuzzy topology on $X$ in Šstak's sense, that is, $\mathcal{T}$ is a mapping from $I^{X}$ to $I$. Take $E=I$ and define $\overline{\mathcal{T}}: E \longrightarrow I^{X}$ as $\overline{\mathcal{T}}(e) \triangleq\{\mu$ : $\mathcal{T}(\mu) \geq e\}$ which is levelwise fuzzy topology of $\mathcal{T}$ in Chang's sense, for each $e \in I$. However, it is well known that each Chang's fuzzy topology can be considered as Šostak fuzzy topology by using fuzzifying method. Hence, $\mathcal{T}(e)$ satisfies (O1), (O2) and (O3).

According to this definition and by using the decomposition theorem of fuzzy sets [12], if we know the resulting fuzzy soft topology, then we can find the first fuzzy topology. Therefore, we can say that a fuzzy topology can be uniquely represented as a fuzzy soft topology.

3.2. Definition. Let $(X, \tau)$ and $\left(Y, \tau^{*}\right)$ be fuzzy soft topological spaces. A fuzzy soft mapping $\varphi_{\psi}$ from $\widetilde{(X, E)}$ into $\widetilde{(Y, F)}$ is called a fuzzy soft continuous map if $\tau_{e}\left(\varphi_{\psi}^{-1}\left(g_{B}\right)\right) \geq \tau_{\psi(e)}^{*}\left(g_{B}\right)$ for all $g_{B} \in \widetilde{(Y, F)}, e \in E$.

The category of fuzzy soft topological spaces and fuzzy soft continuous mappings is denoted by FSTOP.

3.3. Proposition. Let $\left\{\tau_{k}\right\}_{k \in \Gamma}$ be a family of fuzzy soft topologies on $X$. Then $\tau=$ $\bigwedge_{k \in \Gamma} \tau_{k}$ is also a fuzzy soft topology on $X$, where $\tau_{e}\left(f_{A}\right)=\bigwedge_{k \in \Gamma}\left(\tau_{k}\right)_{e}\left(f_{A}\right), \forall e \in$ $E, f_{A} \in \widetilde{(X, E)}$.

Proof. It is straightforward and therefore is omitted.

3.4. Definition. A mapping $\eta: E \longrightarrow[0,1]^{(\widetilde{X, E)}}$ is called a fuzzy soft cotopology on $X$ if it satisfies the following conditions for each $e \in E$ :

(C1) $\eta_{e}(\Phi)=\eta_{e}(\widetilde{E})=1$.

(C2) $\eta_{e}\left(f_{A} \sqcup g_{B}\right) \geq \eta_{e}\left(f_{A}\right) \wedge \eta_{e}\left(g_{B}\right), \quad \forall f_{A}, g_{B} \in \widetilde{(X, E)}$.

(C3) $\eta_{e}\left(\prod_{i \in \Delta}\left(f_{A}\right)_{i}\right) \geq \bigwedge_{i \in \Delta} \eta_{e}\left(\left(f_{A}\right)_{i}\right), \forall\left(f_{A}\right)_{i} \in \widetilde{(X, E)}, i \in \Delta$.

The pair $(X, \eta)$ is called a fuzzy soft cotopological space.

Let $\tau$ be a fuzzy soft topology on $X$, then the mapping $\eta: E \longrightarrow[0,1]^{\widetilde{X, E)}}$ defined by $\eta_{e}\left(f_{A}\right)=\tau_{e}\left(f_{A}^{c}\right), \forall e \in E$ is a fuzzy soft cotopology on $X$. Let $\eta$ be a fuzzy soft cotopology on $X$, then the mapping $\tau: E \longrightarrow[0,1]^{(X, E)}$ defined by $\tau_{e}\left(f_{A}\right)=\eta_{e}\left(f_{A}^{c}\right), \forall e \in E$, is a fuzzy soft topology on $X$.

3.5. Definition. A mapping $\beta: E \longrightarrow[0,1]^{\widetilde{X, E)}}$ is called a fuzzy soft base on $X$ if it satisfies the following conditions for each $e \in E$ :

(B1) $\beta_{e}(\Phi)=\beta_{e}(\widetilde{E})=1$.

(B2) $\beta_{e}\left(f_{A} \sqcap g_{B}\right) \geq \beta_{e}\left(f_{A}\right) \wedge \beta_{e}\left(g_{B}\right), \quad \forall f_{A}, g_{B} \in \widetilde{(X, E)}$.

3.6. Theorem. Let $\beta$ be a fuzzy soft base on $X$. Define a map $\tau_{\beta}: E \longrightarrow[0,1]^{\widetilde{(X, E)}}$ as follows: 


$$
\left(\tau_{\beta}\right)_{e}\left(f_{A}\right)=\bigvee\left\{\bigwedge_{j \in \Lambda} \beta_{e}\left(\left(f_{A}\right)_{j}\right) \mid f_{A}=\bigsqcup_{j \in \Lambda}\left(f_{A}\right)_{j}\right\}, \quad \forall e \in E .
$$

Then $\tau_{\beta}$ is the coarsest fuzzy soft topology on $X$ for which $\left(\tau_{\beta}\right)_{e}\left(f_{A}\right) \geq \beta_{e}\left(f_{A}\right)$, for all $e \in E, f_{A} \in \widetilde{(X, E)}$.

Proof. (O1) It is trivial from the definition of $\tau_{\beta}$.

(O2) Let $e \in E$. For all families $\left\{\left(f_{A}\right)_{j} \mid f_{A}=\bigsqcup_{j \in \Lambda}\left(f_{A}\right)_{j}\right\}$ and $\left\{\left(g_{B}\right)_{k} \mid g_{B}=\right.$ $\left.\bigsqcup_{k \in \Gamma}\left(g_{B}\right)_{k}\right\}$, there exists a family $\left\{\left(f_{A}\right)_{j} \sqcap\left(g_{B}\right)_{k}\right\}$ such that

$$
f_{A} \sqcap g_{B}=\left(\bigsqcup_{j \in \Lambda}\left(f_{A}\right)_{j}\right) \sqcap\left(\bigsqcup_{k \in \Gamma}\left(g_{B}\right)_{k}\right)=\bigsqcup_{j \in \Lambda, k \in \Gamma}\left(\left(f_{A}\right)_{j} \sqcap\left(g_{B}\right)_{k}\right) .
$$

It implies the followings:

$$
\begin{aligned}
\left(\tau_{\beta}\right)_{e}\left(f_{A} \sqcap g_{B}\right) & \geq \bigwedge_{j \in \Lambda, k \in \Gamma} \beta_{e}\left(\left(f_{A}\right)_{j} \sqcap\left(g_{B}\right)_{k}\right) \\
& \geq \bigwedge_{j \in \Lambda, k \in \Gamma}\left(\beta_{e}\left(\left(f_{A}\right)_{j}\right) \wedge \beta_{e}\left(\left(g_{B}\right)_{k}\right)\right) \\
& \geq\left(\bigwedge_{j \in \Lambda} \beta_{e}\left(\left(f_{A}\right)_{j}\right)\right) \wedge\left(\bigwedge_{k \in \Gamma} \beta_{e}\left(\left(g_{B}\right)_{k}\right)\right) \\
& \geq\left(\tau_{\beta}\right)_{e}\left(f_{A}\right) \wedge\left(\tau_{\beta}\right)_{e}\left(g_{B}\right) .
\end{aligned}
$$

(O3) Let $e \in E$ and $\wp_{i}$ be the collection of all index sets $K_{i}$ such that $\left\{\left(f_{A}\right)_{i_{k}} \in\right.$ $\left.\widetilde{(X, E)} \mid\left(f_{A}\right)_{i}=\bigsqcup_{k \in K_{i}}\left(f_{A}\right)_{i_{k}}\right\}$ with $f_{A}=\bigsqcup_{i \in \Gamma}\left(f_{A}\right)_{i}=\bigsqcup_{i \in \Gamma} \bigsqcup_{k \in K_{i}}\left(f_{A}\right)_{i_{k}}$. For each $i \in \Gamma$ and each $\Psi \in \Pi_{i \in \Gamma \wp_{i}}$ with $\Psi(i)=K_{i}$, we have $\bigwedge_{i \in \Gamma}\left(\bigwedge_{k \in K_{i}} \beta_{e}\left(\left(f_{A}\right)_{i_{k}}\right)\right)$

Put $a_{i, \Psi_{i}}=\bigwedge_{k \in K_{i}}\left(\beta_{e}\left(\left(f_{A}\right)_{i_{k}}\right)\right)$. Then we have the following:

$$
\begin{aligned}
\left(\tau_{\beta}\right)_{e}\left(f_{A}\right) & \geq \bigvee_{\Psi \in \Pi_{i \in \Gamma \wp_{i}}}\left(\bigwedge_{i \in \Gamma} a_{i, \Psi(i)}\right) \\
& =\bigwedge_{i \in \Gamma}\left(\bigvee_{M_{i} \in \wp_{i}} a_{i, M_{i}}\right) \\
& =\bigwedge_{i \in \Gamma}\left(\bigvee_{M_{i} \in \wp_{i}}\left(\bigwedge_{m \in M_{i}}\left(\beta_{e}\left(\left(f_{A}\right)_{i_{m}}\right)\right)\right)\right) \\
& =\bigwedge_{i \in \Gamma}\left(\tau_{\beta}\right)_{e}\left(\left(f_{A}\right)_{i}\right) .
\end{aligned}
$$

Thus, $\tau_{\beta}$ is a fuzzy soft topology on $X$. Let $\tau \sqsupseteq \beta$, then for every $e \in E$ and $f_{A}=\bigsqcup_{j \in \Lambda}\left(f_{A}\right)_{j}$, we have

$$
\tau_{e}\left(f_{A}\right) \geq \bigwedge_{j \in \Lambda} \tau_{e}\left(\left(f_{A}\right)_{j}\right) \geq \bigwedge_{j \in \Lambda} \beta_{e}\left(\left(f_{A}\right)_{j}\right) .
$$


If we take supremum over the family $\left\{\left(f_{A}\right)_{j} \in \widetilde{(X, E)} \mid f_{A}=\bigsqcup_{j \in \Lambda}\left(f_{A}\right)_{j}\right\}$, then we obtain that $\tau \sqsupseteq \tau_{\beta}$.

3.7. Lemma. Let $\tau$ be a fuzzy soft topology on $X$ and $\beta$ be a fuzzy soft base on $Y$. Then a fuzzy soft mapping $\varphi_{\psi}$ from $\widetilde{(X, E)}$ into $\widetilde{(Y, F)}$ is fuzzy soft continuous if and only if $\tau_{e}\left(\varphi_{\psi}^{-1}\left(g_{B}\right)\right) \geq \beta_{\psi(e)}\left(g_{B}\right)$, for each $e \in E, g_{B} \in \widetilde{(Y, F)}$.

Proof. $(\Rightarrow)$ Let $\varphi_{\psi}:(X, \tau) \longrightarrow\left(Y, \tau_{\beta}\right)$ be a fuzzy soft continuous mapping and $g_{B} \in \widehat{(Y, F)}$. Then,

$$
\tau_{e}\left(\varphi_{\psi}^{-1}\left(g_{B}\right)\right) \geq\left(\tau_{\beta}\right)_{\psi(e)}\left(g_{B}\right) \geq \beta_{\psi(e)}\left(g_{B}\right) .
$$

$(\Leftarrow)$ Let $\tau_{e}\left(\varphi_{\psi}^{-1}\left(g_{B}\right)\right) \geq \beta_{\psi(e)}\left(g_{B}\right)$, for each $g_{B} \in \widetilde{(Y, F)}$. Let $h_{C} \in \widetilde{(Y, F)}$. For every family of $\left\{\left(h_{C}\right)_{j} \in \widetilde{(Y, F)} \mid h_{C}=\bigsqcup_{j \in \Gamma}\left(h_{C}\right)_{j}\right\}$, we have

$$
\begin{aligned}
\tau_{e}\left(\varphi_{\psi}^{-1}\left(h_{C}\right)\right) & =\tau_{e}\left(\varphi_{\psi}^{-1}\left(\bigsqcup_{j \in \Gamma}\left(h_{C}\right)_{j}\right)\right) \\
& =\tau_{e}\left(\bigsqcup_{j \in \Gamma} \varphi_{\psi}^{-1}\left(\left(h_{C}\right)_{j}\right)\right) \\
& \geq \bigwedge_{j \in \Gamma} \tau_{e}\left(\varphi_{\psi}^{-1}\left(\left(h_{C}\right)_{j}\right)\right) \\
& \geq \bigwedge_{j \in \Gamma} \beta_{\psi(e)}\left(\left(h_{C}\right)_{j}\right) .
\end{aligned}
$$

If we take supremum over the family of $\left\{\left(h_{C}\right)_{j} \in \widetilde{(Y, F)} \mid h_{C}=\bigsqcup_{j \in \Gamma}\left(h_{C}\right)_{j}\right\}$, we obtain

$$
\tau_{e}\left(\varphi_{\psi}^{-1}\left(h_{C}\right)\right) \geq\left(\tau_{\beta}\right)_{\psi(e)}\left(h_{C}\right) .
$$

3.8. Theorem. Let $\left\{\left(X_{i},\left(\tau_{i}\right)_{E_{i}}\right)\right\}_{i \in \Gamma}$ be a family of fuzzy soft topological spaces, $X$ be a set, $E$ be a parameter set and for each $i \in \Gamma, \varphi_{i}: X \rightarrow X_{i}$ and $\psi_{i}: E \rightarrow E_{i}$ be maps. Define $\beta: E \rightarrow[0,1]^{(X, E)}$ on $X$ by:

$$
\beta_{e}\left(f_{A}\right)=\bigvee\left\{\bigwedge_{j=1}^{n}\left(\tau_{k_{j}}\right)_{\psi_{k_{j}}(e)}\left(\left(f_{A}\right)_{k_{j}}\right) \mid f_{A}=\prod_{j=1}^{n}\left(\varphi_{\psi}\right)_{k_{j}}^{-1}\left(\left(f_{A}\right)_{k_{j}}\right)\right\},
$$

where $\bigvee$ is taken over all finite subsets $K=\left\{k_{1}, k_{2}, \ldots, k_{n}\right\} \subset \Gamma$. Then,

(1) $\beta$ is a fuzzy soft base on $X$.

(2) The fuzzy soft topology $\tau_{\beta}$ generated by $\beta$ is the coarsest fuzzy soft topology on $X$ for which all $\left(\varphi_{\psi}\right)_{i}, i \in \Gamma$ are fuzzy soft continuous maps.

(3) A map $\varphi_{\psi}:\left(Y, \delta_{F}\right) \rightarrow\left(X,\left(\tau_{\beta}\right)_{E}\right)$ is fuzzy soft continuous iff for each $i \in \Gamma$, $\left(\varphi_{\psi}\right)_{i} \circ \varphi_{\psi}:\left(Y, \delta_{F}\right) \rightarrow\left(X_{i},\left(\tau_{i}\right)_{E_{i}}\right)$ is a fuzzy soft continuous map.

Proof. (1) (B1) Since $f_{A}=\left(\varphi_{\psi}\right)_{i}^{-1}\left(f_{A}\right)$ for each $f_{A} \in\{\Phi, \widetilde{E}\}, \beta_{e}(\Phi)=\beta_{e}(\widetilde{E})=1$, for each $e \in E$. 
(B2) For all finite subsets $K=\left\{k_{1}, k_{2}, \ldots, k_{n}\right\}$ and $J=\left\{j_{1}, j_{2}, \ldots, j_{m}\right\}$ of $\Gamma$ such that $f_{A}=\prod_{i=1}^{n}\left(\varphi_{\psi}\right)_{k_{i}}^{-1}\left(\left(f_{A}\right)_{k_{i}}\right)$ and $g_{B}=\prod_{i=1}^{m}\left(\varphi_{\psi}\right)_{j_{i}}^{-1}\left(\left(g_{B}\right)_{j_{i}}\right)$, we have $f_{A} \sqcap g_{B}=$ $\left(\prod_{i=1}^{n}\left(\varphi_{\psi}\right)_{k_{i}}^{-1}\left(\left(f_{A}\right)_{k_{i}}\right)\right) \sqcap\left(\prod_{i=1}^{m}\left(\varphi_{\psi}\right)_{j_{i}}^{-1}\left(\left(g_{B}\right)_{j_{i}}\right)\right)$.

Furthermore, we have for each $k \in K \cap J$,

$$
\left(\varphi_{\psi}\right)_{k}^{-1}\left(\left(f_{A}\right)_{k}\right) \sqcap\left(\varphi_{\psi}\right)_{k}^{-1}\left(\left(g_{B}\right)_{k}\right)=\left(\varphi_{\psi}\right)_{k}^{-1}\left(\left(f_{A}\right)_{k} \sqcap\left(g_{B}\right)_{k}\right) .
$$

Put $f_{A} \sqcap g_{B}=\prod_{m_{i} \in K \cup J}\left(\varphi_{\psi}\right)_{m_{i}}^{-1}\left(\left(h_{C}\right)_{m_{i}}\right)$ where

$$
\left(h_{C}\right)_{m_{i}}= \begin{cases}\left(f_{A}\right)_{m_{i}}, & \text { if } m_{i} \in K-(K \cap J) ; \\ \left(g_{B}\right)_{m_{i}}, & \text { if } m_{i} \in J-(K \cap J) ; \\ \left(f_{A}\right)_{m_{i}} \sqcap\left(g_{B}\right)_{m_{i}}, & \text { if } m_{i} \in(K \cap J) .\end{cases}
$$

So we have

$$
\begin{aligned}
\beta_{e}\left(\left(f_{A}\right) \sqcap\left(g_{B}\right)\right) & \geq \bigwedge_{j \in K \cup J}\left(\tau_{j}\right)_{\psi_{j}(e)}\left(\left(h_{C}\right)_{j}\right) \\
& \geq\left(\bigwedge_{i=1}^{n}\left(\tau_{k_{i}}\right)_{\psi_{k_{i}}(e)}\left(\left(f_{A}\right)_{k_{i}}\right)\right) \wedge\left(\bigwedge_{i=1}^{m}\left(\tau_{j_{i}}\right)_{\psi_{j_{i}}(e)}\left(\left(g_{B}\right)_{j_{i}}\right)\right) .
\end{aligned}
$$

If we take supremum over the families $\left\{f_{A}=\prod_{i=1}^{n}\left(\varphi_{\psi}\right)_{k_{i}}^{-1}\left(\left(f_{A}\right)_{k_{i}}\right)\right\}$ and $\left\{g_{B}=\right.$ $\left.\prod_{i=1}^{m}\left(\varphi_{\psi}\right)_{j_{i}}^{-1}\left(\left(g_{B}\right)_{j_{i}}\right)\right\}$, then we have,

$$
\beta_{e}\left(f_{A} \sqcap g_{B}\right) \geq \beta_{e}\left(f_{A}\right) \wedge \beta_{e}\left(g_{B}\right), \forall e \in E .
$$

(2) For each $\left(f_{A}\right)_{i} \in\left(\widetilde{X_{i}, E_{i}}\right)$, one family $\left\{\left(\varphi_{\psi}\right)_{i}^{-1}\left(\left(f_{A}\right)_{i}\right)\right\}$ and $i \in \Gamma$, we have $\left(\tau_{\beta}\right)_{e}\left(\left(\varphi_{\psi}\right)_{i}^{-1}\left(\left(f_{A}\right)_{i}\right)\right) \geq \beta_{e}\left(\left(\varphi_{\psi}\right)_{i}^{-1}\left(\left(f_{A}\right)_{i}\right)\right) \geq\left(\tau_{i}\right)_{\psi_{i}(e)}\left(\left(f_{A}\right)_{i}\right)$, for each $e \in E$.

Therefore, for all $i \in \Gamma,\left(\varphi_{\psi}\right)_{i}:\left(X,\left(\tau_{\beta}\right)_{E}\right) \longrightarrow\left(X_{i},\left(\tau_{i}\right)_{E_{i}}\right)$ is fuzzy soft continuous.

Let $\left(\varphi_{\psi}\right)_{i}:\left(X, \zeta_{E}\right) \longrightarrow\left(X_{i},\left(\tau_{i}\right)_{E_{i}}\right)$ be fuzzy soft continuous, that is, for each $i \in \Gamma$ and $\left(f_{A}\right)_{i} \in\left(\widetilde{X_{i}, E_{i}}\right), \zeta_{e}\left(\left(\varphi_{\psi}\right)_{i}^{-1}\left(\left(f_{A}\right)_{i}\right)\right) \geq\left(\tau_{i}\right)_{\psi_{i}(e)}\left(\left(f_{A}\right)_{i}\right)$.

For all finite subsets $K=\left\{k_{1}, \ldots, k_{n}\right\}$ of $\Gamma$ such that $f_{A}=\prod_{i=1}^{n}\left(\varphi_{\psi}\right)_{k_{i}}^{-1}\left(\left(f_{A}\right)_{k_{i}}\right)$ we have

$$
\zeta_{e}\left(f_{A}\right) \geq \bigwedge_{i=1}^{n} \zeta_{e}\left(\left(\varphi_{\psi}\right)_{k_{i}}^{-1}\left(\left(f_{A}\right)_{k_{i}}\right)\right) \geq \bigwedge_{i=1}^{n}\left(\tau_{k_{i}}\right)_{\psi_{k_{i}}(e)}\left(\left(f_{A}\right)_{k_{i}}\right) .
$$

It implies $\zeta_{e}\left(f_{A}\right) \geq \beta_{e}\left(f_{A}\right)$, for all $e \in E, f_{A} \in \widetilde{(X, E)}$. By Theorem 3.6, $\zeta \sqsupseteq \tau_{\beta}$. (3) $(\Rightarrow)$ Let $\varphi_{\psi}:\left(Y, \delta_{F}\right) \rightarrow\left(X,\left(\tau_{\beta}\right)_{E}\right)$ be fuzzy soft continuous. For each $i \in \Gamma$ and $\left(f_{A}\right)_{i} \in\left(\widetilde{X_{i}, E_{i}}\right)$ we have 


$$
\begin{gathered}
\delta_{\mathrm{f}}\left(\left(\varphi_{i} \circ \varphi\right)_{\psi_{i} \circ \psi}^{-1}\left(\left(f_{A}\right)_{i}\right)\right)=\delta_{\mathrm{f}}\left(\varphi_{\psi}^{-1}\left(\left(\varphi_{\psi}\right)_{i}^{-1}\left(\left(f_{A}\right)_{i}\right)\right)\right) \geq\left(\tau_{\beta}\right)_{\psi(\mathrm{f})}\left(\left(\varphi_{\psi}\right)_{i}^{-1}\left(\left(f_{A}\right)_{i}\right)\right) \geq \\
\left(\tau_{i}\right)_{\left(\psi_{i} \circ \psi\right)(\mathrm{f})}\left(\left(f_{A}\right)_{i}\right) .
\end{gathered}
$$

Hence, $\left(\varphi_{i} \circ \varphi\right)_{\psi_{i} \circ \psi}:\left(Y, \delta_{F}\right) \rightarrow\left(X_{i},\left(\tau_{i}\right)_{E_{i}}\right)$ is fuzzy soft continuous.

$(\Leftarrow)$ For all finite subsets $K=\left\{k_{1}, \ldots, k_{n}\right\}$ of $\Gamma$ such that $f_{A}=\prod_{i=1}^{n}\left(\varphi_{\psi}\right)_{k_{i}}^{-1}\left(\left(f_{A}\right)_{k_{i}}\right)$, since

$\left(\varphi_{k_{i}} \circ \varphi\right)_{\psi_{k_{i}} \circ \psi}:\left(Y, \delta_{F}\right) \rightarrow\left(X_{k_{i}},\left(\tau_{k_{i}}\right)_{E_{k_{i}}}\right)$ is fuzzy soft continuous, $\delta_{\mathrm{f}}\left(\varphi_{\psi}^{-1}\left(\left(\varphi_{\psi}\right)_{k_{i}}^{-1}\left(\left(f_{A}\right)_{k_{i}}\right)\right)\right) \geq$ $\left(\tau_{k_{i}}\right)_{\left(\psi_{i} \circ \psi\right)(\mathrm{f})}\left(\left(f_{A}\right)_{k_{i}}\right), \forall \mathrm{f} \in F$.

Hence we have

$$
\begin{aligned}
\delta_{\mathrm{f}}\left(\varphi_{\psi}^{-1}\left(f_{A}\right)\right) & =\delta_{\mathrm{f}}\left(\varphi_{\psi}^{-1}\left(\prod_{i=1}^{n}\left(\varphi_{\psi}\right)_{k_{i}}^{-1}\left(\left(f_{A}\right)_{k_{i}}\right)\right)\right) \\
& =\delta_{\mathrm{f}}\left(\prod_{i=1}^{n}\left(\varphi_{\psi}^{-1}\left(\left(\varphi_{\psi}\right)_{k_{i}}^{-1}\left(\left(\left(f_{A}\right)_{k_{i}}\right)\right)\right)\right)\right) \\
& \geq \bigwedge_{i=1}^{n} \delta_{\mathrm{f}}\left(\varphi_{\psi}^{-1}\left(\left(\varphi_{\psi}\right)_{k_{i}}^{-1}\left(\left(\left(f_{A}\right)_{k_{i}}\right)\right)\right)\right) \\
& \geq \bigwedge_{i=1}^{n}\left(\tau_{k_{i}}\right)_{\left(\psi_{k_{i}} \circ \psi\right)(\mathrm{f})}\left(\left(f_{A}\right)_{k_{i}}\right) .
\end{aligned}
$$
F.

This inequality implies that $\delta_{\mathrm{f}}\left(\varphi_{\psi}^{-1}\left(f_{A}\right)\right) \geq \beta_{\psi(\mathrm{f})}\left(f_{A}\right)$ for each $f_{A} \in \widetilde{(X, E)}, \mathrm{f} \in$

By Lemma 3.7, $\varphi_{\psi}:\left(Y, \delta_{F}\right) \rightarrow\left(X,\left(\tau_{\beta}\right)_{E}\right)$ is fuzzy soft continuous.

Let $\left\{\left(X_{i},\left(\tau_{i}\right)_{E_{i}}\right)\right\}_{i \in \Gamma}$ be a family of fuzzy soft topological spaces, $X$ be a set, $E$ be a parameter set and for each $i \in \Gamma, \varphi_{i}: X \rightarrow X_{i}$ and $\psi_{i}: E \rightarrow E_{i}$ be maps. The initial fuzzy soft topology $\tau_{\beta}$ on $X$ is the coarsest fuzzy soft topology on $X$ for which all $\left(\varphi_{\psi}\right)_{i}, i \in \Gamma$ are fuzzy soft continuous maps.

3.9. Definition. [1] A category $\mathbf{C}$ is called a topological category over SET with respect to the usual forgetful functor from $\mathbf{C}$ to SET if it satisfies the following conditions:

(TC1) Existence of initial structures: For any $X$, any class $J$, and any family $\left(\left(X_{j}, \xi_{j}\right)\right)_{j \in J}$ of $\mathbf{C}$-object and any family $\left(f_{j}: X \longrightarrow X_{j}\right)_{j \in J}$ of maps, there exists a unique $\mathbf{C}$-structure $\xi$ on $X$ which is initial with respect to the source $\left(f_{j}: X \longrightarrow\right.$ $\left.\left(X_{j}, \xi_{j}\right)\right)_{j \in J}$, this means that for a $\mathbf{C}$-object $(Y, \eta)$, a map $g:(Y, \eta) \longrightarrow(X, \xi)$ is a $\mathbf{C}$-morphism if and only if for all $j \in J, f_{j} \circ g:(Y, \eta) \longrightarrow\left(X_{j}, \xi_{j}\right)$ is a $\mathbf{C}$-morphism.

(TC2) Fibre smallness: For any set $X$, the $\mathbf{C}$-fibre of $X$, i.e., the class of all $\mathbf{C}$-structure on $X$, which we denote $\mathbf{C}(\mathrm{X})$, is a set.

3.10. Theorem. The category FSTOP is a topological category over SET with respect to the forgetful functor $V: \mathbf{F S T O P} \longrightarrow \mathbf{S E T}$ which is defined by $V\left(X, \tau_{E}\right)=$ $X$ and $V\left(\varphi_{\psi}\right)=\varphi$.

Proof. The proof is straightforward and follows from Theorem 3.8.

3.11. Definition. Let $\left\{\left(X_{i},\left(\tau_{i}\right)_{E_{i}}\right)\right\}_{i \in \Gamma}$ be a family of fuzzy soft topological spaces, for each $i \in \Gamma, E_{i}$ be parameter sets, $X=\prod_{i \in \Gamma} X_{i}$ and $E=\prod_{i \in \Gamma} E_{i}$. Let $p_{i}: X \longrightarrow X_{i}$ and $q_{i}: E \longrightarrow E_{i}$ be projection maps, for all $i \in \Gamma$. The product 
of fuzzy soft topologies $\left(X, \tau_{E}\right)$ with respect to parameter set $E$ is the coarsest fuzzy soft topology on $X$ for which all $\left(p_{q}\right)_{i}, i \in \Gamma$, are fuzzy soft continuous maps.

\section{Conclusion}

In this paper, we have considered the topological structure of fuzzy soft set theory. We have given the definition of fuzzy soft topology $\tau$ which is a mapping from the parameter set $E$ to $[0,1]^{(\widetilde{X, E)}}$ which satisfy the three certain conditions. Since the value of a fuzzy soft set $f_{A}$ under the mapping $\tau_{e}$ gives us the degree of openness of the fuzzy soft set with respect to the parameter $e \in E, \tau_{e}$ can be thought of as a fuzzy soft topology in the sense of Šostak. In this sense, we have introduced fuzzy soft cotopology and given the relations between fuzzy soft topology and fuzzy soft cotopology. Then we have defined fuzzy soft base and by using a fuzzy soft base we have obtained a fuzzy soft topology on the same set. Also, we have introduced an initial fuzzy soft topology and then we have given the definition of product fuzzy soft topology. Further, we have proved that the category of fuzzy soft topological spaces FSTOP is a topological category over SET with respect to the forgetful functor.

\section{References}

[1] J. Adámek, H. Herrlich, G. E. Strecker, Abstract and Concrete Categories, Wiley, New York, 1990.

[2] B. Ahmad, A. Kharal, On Fuzzy Soft Sets, Advances in Fuzzy Systems, Volume 2009, Article ID 586507

[3] H. Aktaş, N. Çağman, Soft sets and soft groups, Information Sciences 177 (13) (2007) 27262735.

[4] K. Atanassov, Intuitionistic Fuzzy Sets, Fuzzy Sets and Systems 64 (2) (1986) 87-96.

[5] A. Aygünoğlu, H. Aygün, Introduction to fuzzy soft groups, Computers and Mathematics with Applications 58 (2009) 1279-1286

[6] F. Feng, Y. B. Jun, X. Zhao, Soft semirings, Computers and Mathematics with Applications 56 (10) (2008) 2621-2628.

[7] Y. B. Jun, Soft BCK/BCI algebras, Computers and Mathematics with Applications 56 (5) (2008) 1408-1413.

[8] Y. B. Jun, C. H. Park, Applications of soft sets in ideal theory of BCK/BCI algebras, Information Sciences 178 (11) (2008) 2466-2475.

[9] Y. B. Jun, K. J. Lee, C. H. Park, Soft set theory applied to ideals in d-algebras, Computers and Mathematics with Applications 57 (3) (2009) 367-378.

[10] Y. B. Jun, K. J. Lee, J. Zhan, Soft p-ideals of soft BCI-algebras, Computers and Mathematics with Applications 58 (10) (2009) 2060-2068.

[11] A. Kharal, B. Ahmad, Mappings on Fuzzy Soft Classes, Advances in Fuzzy Systems, Volume 2009, Article ID 407890

[12] G.J. Klir, B. Yuan, Fuzzy sets and fuzzy logic, Theory and Applications, Prentice-Hall Inc., New Jersey, 1995.

[13] P.K. Maji, R. Biswas, A.R. Roy, Fuzzy soft sets, Journal of fuzzy Mathematics, 9(3) (2001) 589-602.

[14] P. K. Maji, A. R. Roy and R. Biswas, An application of soft sets in a decision making problem, Computers and Mathematics with Applications 44 (8-9) (2002) 1077-1083.

[15] D. Molodtsov, Soft set theory-First results, Computers Mathematics with Appl. 37 (4/5) (1999) 19-31.

[16] Z. Pawlak, Rough Sets, International Journal of Information and Computer Science 11 (1982) 341-356. 
[17] D. Pei, D. Miao, From soft sets to information systems, Granular Computing, 2005 IEEE International Conference on (2) (2005) 617-621.

[18] A.R. Roy, P.K. Maji, A fuzzy soft set theoretic approach to decision making problems, Journal of Computational and Applied Mathematics, 203 (2007) 412-418.

[19] A. P. S̆ostak, On a fuzzy topological structure, Suppl. Rend. Circ. Matem. Palermo, Ser II 11 (1985) 89-103.

[20] Q. M. Sun, Z. L. Zhang, J. Liu, Soft sets and soft modules in: G. Wang, T. Li, J. W. Grzymala-Busse, D. Miao, A. Skowron, Y. Yao (Eds.) Proceedings of the Third International Conference on Rough Sets and Knowledge Technology, RSKT 2008, in: Lecture Notes in Computer Science, vol. 5009, Springer (2008) 403-409.

[21] L. A. Zadeh, Fuzzy Sets, Information and Control 8 (1965) 338-353. 